- Case Report

\title{
Lucio Phenomenon: Sequelae of Neglected Leprosy
}

\author{
Siti Nuryati Che Ya ${ }^{1}$, Rosediani Muhamad ${ }^{1}{ }^{*}$, Rosnani Zakaria' ${ }^{1}$, Azlina Ishak ${ }^{1}$, Wan Noor Hasbee Wan Abdullah ${ }^{2}$ \\ 'Department of Family Medicine, School of Medical Sciences, Universiti Sains Malaysia, Kubang Kerian, Malaysia \\ ${ }^{2}$ Department of Dermatology, Hospital Raja Perempuan Zainab II, Kelantan, Malaysia
}

Lucio phenomenon is a potentially fatal leprosy reaction seen in pure, primitive, and diffuse form of lepromatous leprosy. It is a globally restricted phenomenon that occurs in Mexico and Costa Rica; however, sporadic cases have been reported elsewhere. We report a rare case of a 50-year-old Indonesian, who resided in Malaysia for the past 33 years, which presented with extensive ulcers on all four limbs for 3 weeks, as well as a 16-year history of skin changes and alopecia. Full evaluation established the diagnosis of lepromatous leprosy with Lucio phenomenon. Delay in diagnosis leads to significant disabilities and disease transmission within the community. Therefore, primary care practitioners, even in non-endemic countries, should be wary of this atypical feature of leprosy to prevent complications since it is a highly curable disease.

Keywords: Lucio Phenomenon; Leprosy; Lepromatous Leprosy; Ulcer 


\section{INTRODUCTION}

Lucio leprosy is a pure, primitive, and diffuse non-nodular form of lepromatous leprosy. ${ }^{1)}$ Lucio's phenomenon is a rare, aggressive, and occasionally fatal type-II lepra reaction termed "erythema necroticans" that occurs in the course of Lucio leprosy., ${ }^{2,3)}$ It was first described in 1852 by Lucio and Alvarado as a necrotizing skin reaction affecting patients with non-nodular lepromatous leprosy in Mexico. ${ }^{1,2,45}$ A decade later, it was so named in 1948 by Latapi and Zamora, and they further elaborated upon its definition describing it as histopathologic vasculitis with exclusive occurrence in untreated, diffuse non-nodular lepromatous leprosy. ${ }^{1,5)}$

Lucio phenomenon is clinically characterized by necrotic hemorrhagic lesions on the extremities and trunk. It has been reported primarily in Mexico and Costa Rica. ${ }^{1)}$ However, sporadic cases have been reported in Brazil, Argentina, India, and Singapore. ${ }^{2-4,6)}$ To the best of our knowledge, the first Lucio phenomenon case series in Malaysia was reported in 2009, where one out of three patients succumbed to the disease. ${ }^{1)}$ Our patient, an Indonesian, has been a resident of Malaysia for the past 33 years and survived the ordeal. Even though the disease is highly treatable, a delay in his diagnosis led to permanent disabilities and transmission within his family.

\section{CASE REPORT}

A 50-year-old Indonesian gentleman who worked as a laborer at a remote local estate in Malaysia for the preceding 33 years presented with extensive ulcers involving the upper and lower limbs. The ulcers were painful, with rapid progression over 3 weeks. It was associated with fever and rigor. He visited a local general practitioner on day 3 of the illness and was prescribed oral antibiotics and a topical antifungal. In

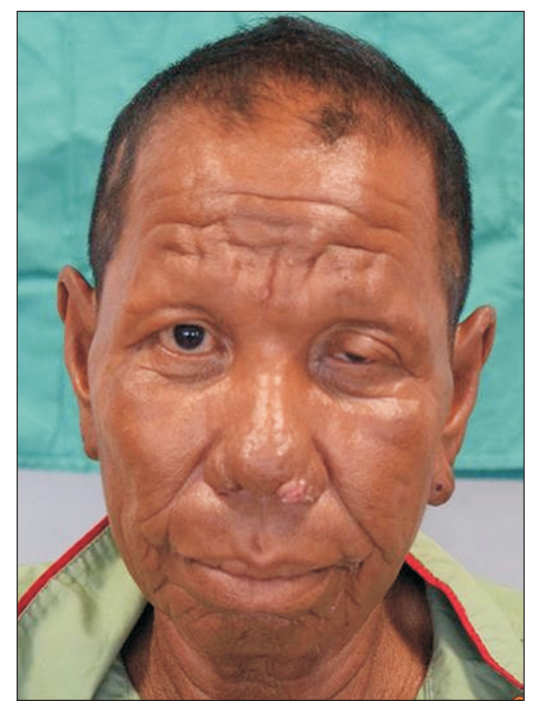

Figure 1. Waxy infiltration with leonine facies, madarosis, rhinophyma, granulomatous lesion over left alae nasi, and left third nerve palsy. Written informed consent for publication of this image was obtained from the patient. retrospect, diffuse skin changes, nail loss, elongated ears, and patchy alopecia were noted since 2002; however, he did not seek treatment for these symptoms. Regarding his social history, he migrated to Malaysia in 1985 with 2-yearly short visits to Indonesia.

Physical examination revealed that the patient was febrile and hemodynamically stable. The skin was darkened with hyperesthesia and anhidrosis. There was a waxy infiltration with leonine facies, madarosis, rhinophyma with granulomatous lesion over the left alae nasi, and left third-nerve palsy (Figure 1). The earlobes were thickened and elongated (Figure 2). There were widely disseminated necrotic ulcerated hemorrhagic ulcers with serous discharge on both of the lower

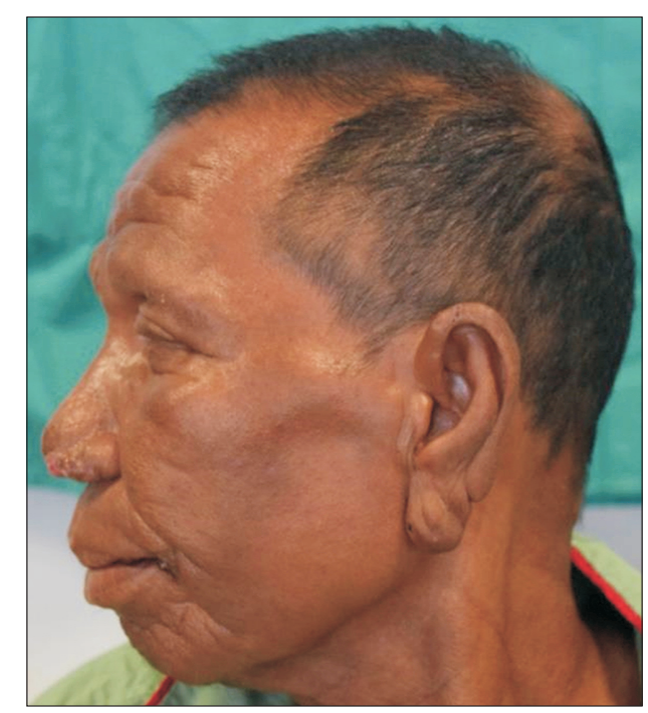

Figure 2. Thickened and elongated earlobes. Written informed consent for publication of this image was obtained from the patient.

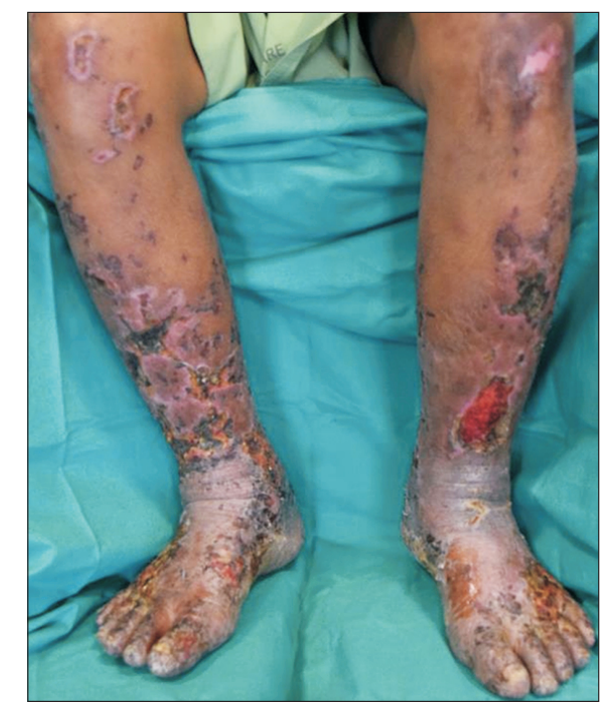

Figure 3. Necrotic ulcerated hemorrhagic ulcers with serous discharge on both lower limbs. Written informed consent for publication of this image was obtained from the patient. 
limbs. Areas of necrotic eschar and achromic scars were noted (Figure 3). Other findings noted were dactylitis with sloughing in acral sites involving all fingers (Figure 4). Axillary nerves were found to be enlarged upon palpation.

The hemogram showed microcytic hypochromic anemia (hemoglobin $10.1 \mathrm{~g} / \mathrm{dL}$, mean corpuscular volume $67 \mathrm{fL}$, mean corpuscular hemoglobin $21 \mathrm{pg})$, normal white blood cell $\left(11.6 \times 10^{3} / \mathrm{uL}\right.$; predominantly neutrophils $71 \%)$, and normal platelet counts $(336 \times 10)$. Iron studies showed reduced serum iron $(4.8 \mathrm{mcmol} / \mathrm{L})$, and the complete blood count revealed hypochromic microcytic anemia secondary to iron deficiency anemia or chronic disease. Moreover, we observed hypoalbuminemia ( $32 \mathrm{~g} / \mathrm{L}$ ) with normal liver enzymes. Renal function and glucose-6-phosphate-dehydrogenase levels were normal. The swab culture from the right leg grew Pseudomonas aeruginosa.

Slit skin smear from the earlobes indicated a bacterial index of 4.0

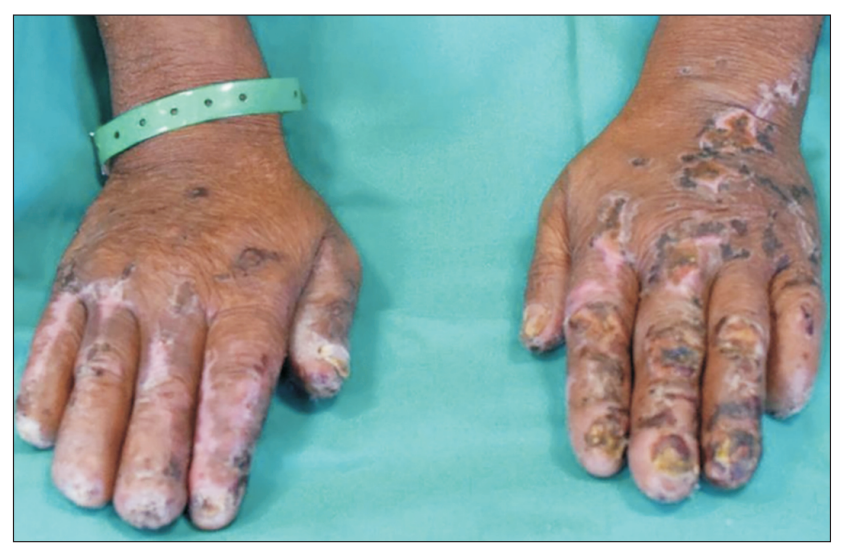

Figure 4. Dactylitis with sloughing of all fingers. Written informed consent for publication of this image was obtained from the patient. (average, 10-1000/field) and a morphological index of 3.0. A skin biopsy of the right chest showed infiltration by neutrophils associated with leukocytoclasis, fibrinoid necrosis of blood vessel walls, and extravasation of red blood cells (Figure 5A, B). The Ziehl-Neelson and Wade Fite stains for acid-fast bacilli were positive (Figure 5C).

A diagnosis of lepromatous leprosy (Ridley-Jopling classification) with Lucio phenomenon complicated with bacterial infection was made. According to World Health Organisation (WHO) guidelines, multidrug therapy (MDT) for multibacillary leprosy was initiated with rifampicin (600 mg/mo), dapsone (100 mg/d), and clofazimine (50 $\mathrm{mg} / \mathrm{d}$ and $300 \mathrm{mg} / \mathrm{mo}$ ) and continued for 18 months in combination with Prednisolone $0.5 \mathrm{mg} / \mathrm{kg} / \mathrm{d}$ for 2 weeks, which was then tapered off due to potential reactions. Intravenous Cefuroxime was also started to cover the Pseudomonas infection. Wound debridement was performed, followed by daily dressing with potassium permanganate solution. Subsequently, the patient developed a neuropathic ulcer on his right second toe which then became infected and had to undergo rays amputation. He completed MDT treatment for 18 months based on modified WHO guidelines and survived. His slit skin smear test on bacterial and morphological indexes was 0.0 after MDT. Clinically, he had a grade 2 disability based on the WHO 1988 disability grading, which consisted of permanent anesthesia and disfigurement of all fingers. Contact screening was done for the family members and his daughter was diagnosed as having paucibacillary leprosy and was on treatment after the screening. The patient completed treatment and survived but ended up with permanent disfigurements. The patient provided written informed consent for the publication of clinical details and images.
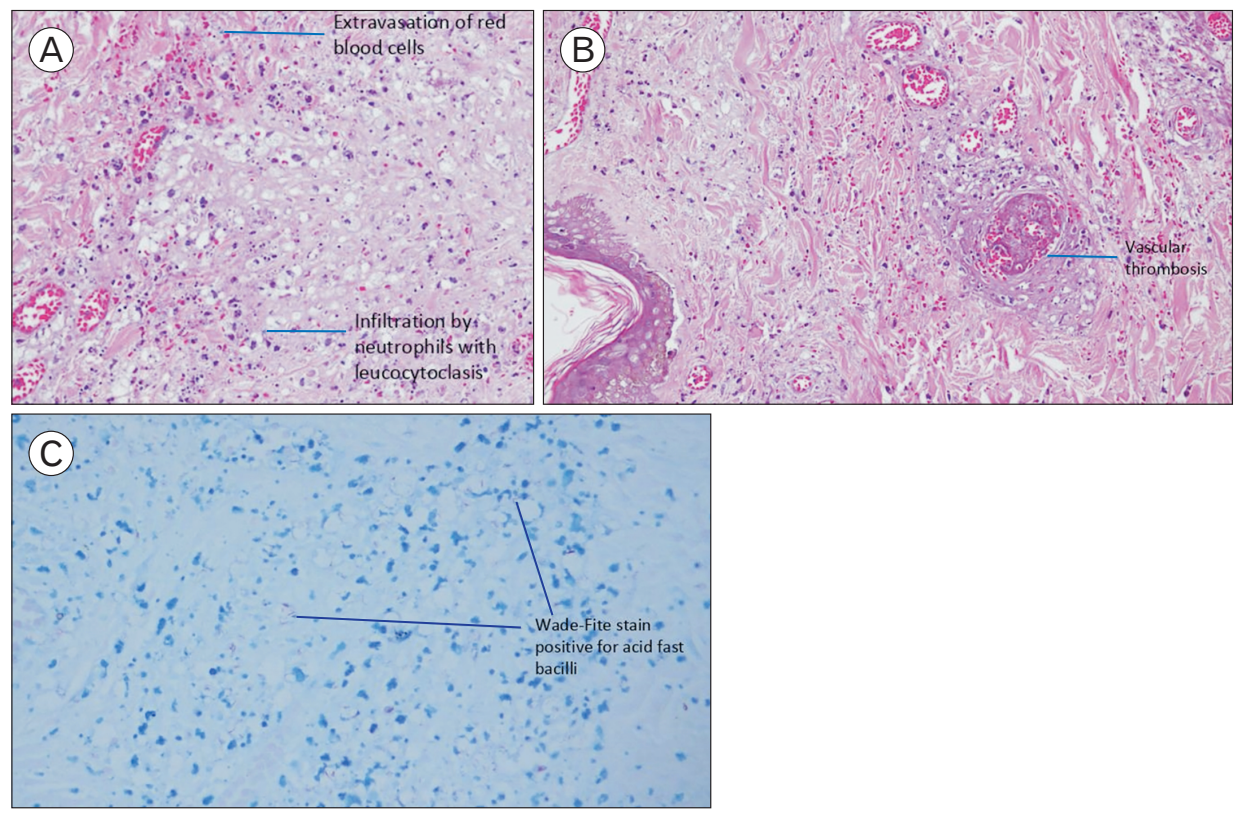

Figure 5. A skin biopsy showed (A) filtration by neutrophils associated with leucocytoclasis and extravasation of red blood cells and (B) vascular thrombosis (Ziehl-Neelson stain, original magnification $[\mathrm{OM}] \times 400)$. (C) positive for acid fast bacilli (Wade Fite stain, OM $\times 400)$. 


\section{DISCUSSION}

Leprosy patients tend to have reactions based on the pattern of the immune response mounted against the bacilli, and the reactions are further subcategorized based on the mechanism. ${ }^{6}$ Lucio phenomenon occurs in the lepromatous version of the illness, where the unresponsiveness of the immune system allows unrestricted proliferation of Mycobacterium sp. to manifest distinct clinic-pathologic features towards the diffuse end of the disease spectrum. ${ }^{6}$ Monteiro et al. ${ }^{2)}$ suggested that the histopathologic characteristics of this phenomenon is due to the inflammatory reactions and changes in the coagulation systems causing vascular thrombosis, ischemia, infarction, and tissue necrosis. Lucio phenomenon is characterized by the existence of immune complexes, necrotizing vasculitis on superficial and mediumsized vessels, diffuse infiltration of the skin, dermal necrosis, and sometimes systemic symptoms. ${ }^{7)}$

The term "Lucio phenomenon" should strictly be used when there is a correlation between clinical and anatomic findings and in accordance with strict clinical criteria. Our patient met the three criteria that define Lucio's phenomenon according to the international literature: skin ulceration, vascular thrombosis, and invasion of blood vessels by Hansen bacilli. ${ }^{4)}$ The predilection of the diffuse lesion in the Lucio phenomenon is mainly extremities, which may include nodules and heal with atrophic stellate scars, as seen in our patient. ${ }^{4)}$

In a series of 30 patients studied by Rea and Jerskey, ${ }^{8)}$ only four were diagnosed with leprosy before the development of the Lucio phenomenon. It may not be easily recognized because of its rarity in non-endemic countries, as well as its similarity with some other manifestations of rheumatic disease and other causes of vasculitis. ${ }^{4)}$ In a study conducted in Malaysia involving 27 cases between 2008-2013, Kwan et al. ${ }^{9)}$ reported that up to $44 \%$ of cases seen initially in a primary care setup were misdiagnosed. Our patient was treated as having a fungal infection that was superimposed with a bacterial infection on day 3 of illness by the local primary care doctor despite having common signs of madarosis, thickened earlobes, and sloughing of the fingernails. Education is essential among health professionals as WHO has recognized leprosy as one of the neglected tropical disease. ${ }^{10)}$ Other differential diagnoses that could mimic the Lucio phenomenon include vasculitides, thrombotic vasculopathy, pyoderma gangrenosum, or connective tissue disease.

The common manifestation of lepromatous leprosy includes the loss of eyebrows (100\%), the loss of eyelashes, and rarely, the loss of body and scalp hair, the thickening of facial skin and earlobes, a waxy infiltrated myxedematous appearance of the skin, peripheral neuropathy, and destructive rhinitis. ${ }^{3)}$ The cases from non-endemic countries have mostly presented with painless progressive necrotic ulcers and systemic symptoms. ${ }^{3,6)}$ Choon and Tey ${ }^{1)}$ also reported atypical features of hepatosplenomegaly and generalized lymphadenopathy in one of the case series in Malaysia. Choon and Tey ${ }^{1)}$ and Curi et al. ${ }^{4)}$ reported that the Lucio phenomenon commonly appears between 1 and 3 years after the manifestation of the disease. On the contrary, our pa- tient had the classical manifestation onset 16 years ago before he sought treatment when the Lucio phenomenon developed. It was postulated that without the familiar nodules associated with lepromatous leprosy, the subtle clinical signs are easily ignored by the patients until more evident findings of the Lucio phenomenon occur. The WHO emphasized strengthening patient and community awareness of leprosy with the aim to increase self-referrals as one of the pillars in the Global Leprosy Strategy. ${ }^{10)}$

The Lucio phenomenon frequently occurs in the untreated, diffuse form of lepromatous leprosy due to uninhibited multiplication of $\mathrm{Ba}$ cilli, as seen in our patient. However, it may also be seen in partially treated lepromatous leprosy. ${ }^{2,6)}$ The prognostic factors and exact mortality rate cannot be predicted because of the small number of cases reported in the literature. ${ }^{6)}$ The response to the treatment of the Lucio phenomenon appears to be poor, with a few fatal cases reported, with cause of death being mainly secondary to sepsis. ${ }^{1,3,4)}$ The Lucio phenomenon necessitates the usual multidrug regiment as an effective first-line treatment with systemic corticosteroid to be included in the regimen for severe situations. ${ }^{6}$ Our patient had a secondary infection with Pseudomonas aeruginosa but survived with prompt treatment, including wound debridement and intravenous antibiotics.

Our case highlighted the diagnostic dilemma of lepromatous leprosy in non-endemic areas that resulted in loss of treatment time with significant morbidities and transmission. It may masquerade as many conditions and should be included in the differential diagnoses in all patients presenting with extensive leg purpura and ulcerations. It is imminent to train the front-liners in non-endemic countries to be vigilant and have a high index of suspicion to prevent unfavorable outcomes since it is highly curable. Timely diagnosis with the help of slit skin smears and skin biopsies will allow for early institution of appropriate therapy. On the other hand, the levels of community information and education about this neglected tropical disease need to be increased.

\section{CONFLICT OF INTEREST}

No potential conflict of interest relevant to this article was reported.

\section{ACKNOWLEDGMENTS}

We would like to thank Department of Dermatology of Hospital Raja Perempuan Zainab II for the support.

\section{ORCID}

Siti Nuryati Che Ya: https://orcid.org/0000-0001-9497-0830 Rosediani Muhamad: https://orcid.org/0000-0002-8393-7899 Rosnani Zakaria: https://orcid.org/0000-0002-7093-8278 Azlina Ishak: https://orcid.org/0000-0001-7289-8460 Wan Noor Hasbee: https://orcid.org/0000-0001-5610-7833 


\section{REFERENCES}

1. Choon SE, Tey KE. Lucio's phenomenon: a report of three cases seen in Johor, Malaysia. Int J Dermatol 2009;48:984-8.

2. Monteiro R, Abreu MA, Tiezzi MG, Roncada EV, Oliveira CC, Ortigosa LC. Lucio's phenomenon: another case reported in Brazil. An Bras Dermatol 2012;87:296-300.

3. Ang P, Tay YK, Ng SK, Seow CS. Fatal Lucio's phenomenon in 2 patients with previously undiagnosed leprosy. J Am Acad Dermatol 2003; 48:958-61.

4. Curi PF, Villaroel JS, Migliore N, Albertengo A, Aquino ML, Ceccato F, et al. Lucio's phenomenon: report of five cases. Clin Rheumatol 2016; 35:1397-401.

5. Vera-Cabrera L, Escalante-Fuentes WG, Gomez-Flores M, OcampoCandiani J, Busso P, Singh P, et al. Case of diffuse lepromatous leprosy associated with "Mycobacterium lepromatosis". J Clin Microbiol 2011;
49:4366-8.

6. Kaur C, Thami GP, Mohan H. Lucio phenomenon and Lucio leprosy. Clin Exp Dermatol 2005;30:525-7.

7. Velarde-Felix JS, Alvarado-Villa G, Vera-Cabrera L. “Lucio's phenomenon" associated with mycobacterium lepromatosis. Am J Trop Med Hyg 2016;94:483-4.

8. Rea TH, Jerskey RS. Clinical and histologic variations among thirty patients with Lucio's phenomenon and pure and primitive diffuse lepromatosis (Latapi's lepromatosis). Int J Lepr Other Mycobact Dis 2005; 73:169-88.

9. Kwan Z, Pailoor J, Tan LL, Robinson S, Wong SM, Ismail R. Leprosy: an imported disease. Lepr Rev 2014;85:170-6.

10. World Health Organization. Global Leprosy Strategy 2016-2020: accelerating towards a leprosy-free world. New Delhi: World Health Organization India; 2016. 